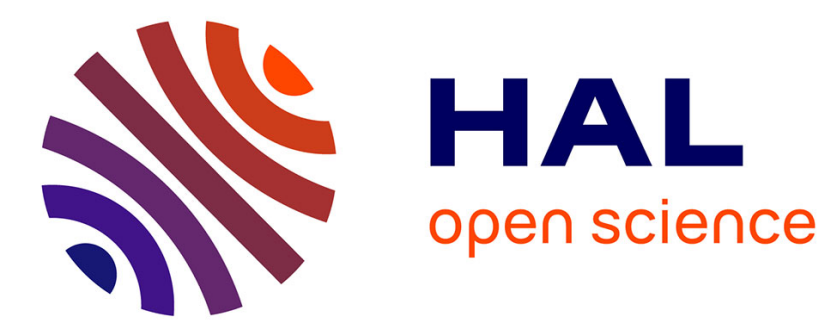

\title{
Pauvreté et accès à l'éducation dans les périphéries d'Oran
}

\author{
Fafa Rebouha, Pascal Pochet
}

\section{To cite this version:}

Fafa Rebouha, Pascal Pochet. Pauvreté et accès à l'éducation dans les périphéries d'Oran. Autrepart - Revue de sciences sociales au Sud, 2011, 2011/3 (59), pp.181-198. halshs-00666342

\section{HAL Id: halshs-00666342 \\ https://shs.hal.science/halshs-00666342}

Submitted on 28 Sep 2014

HAL is a multi-disciplinary open access archive for the deposit and dissemination of scientific research documents, whether they are published or not. The documents may come from teaching and research institutions in France or abroad, or from public or private research centers.
L'archive ouverte pluridisciplinaire HAL, est destinée au dépôt et à la diffusion de documents scientifiques de niveau recherche, publiés ou non, émanant des établissements d'enseignement et de recherche français ou étrangers, des laboratoires publics ou privés. 
Rebouha F., Pochet P. (2011), Pauvreté et accès à l'éducation dans les périphéries d'Oran, Autrepart, n59, pp. 181-198.

\title{
Pauvreté et accès à l'éducation dans les périphéries d'Oran
}

\author{
Fafa Rebouha ${ }^{*}$, Pascal Pochet ${ }^{* *}$
}

Résumé : En dépit de nets progrès en matière de scolarisation, les inégalités sociales et spatiales d'accès à l'éducation demeurent marquées en Algérie. Dans les périphéries non loties d'Oran, souséquipées en services collectifs et relativement enclavées, se concentrent des ménages pauvres qui rencontrent des difficultés importantes pour scolariser leurs enfants. L'objet de cet article est de décrire ces difficultés et d'en rechercher l'origine dans les facteurs liés à l'offre scolaire (répartition spatiale et éloignement, qualité, coût de l'éducation), comme dans ceux qui sont liés aux caractéristiques des ménages. Après une présentation de la répartition de l'offre scolaire, les auteurs mènent une analyse sur les inégalités d'accès à l'éducation basée sur une série d'entretiens qualitatifs menés dans huit quartiers de la périphérie d'Oran.

Mots-clés : offre d'éducation - accès à l'école - pauvreté - périphéries non loties - distance transport collectif - approche qualitative - Oran - Algérie.

\section{Poverty and Access to Education in the Peripheries of Oran}

\begin{abstract}
Despite significant progress in schooling, social and spatial inequalities in access to education remain important in Algeria. The un-plotted peripheries of Oran, underequipped with urban services and relatively isolated, concentrate poor households facing important difficulties to educate their children. The purpose of this paper is to describe these difficulties, and look for their origin in the characteristics of school provision (spatial distribution and distance, quality, education cost) as well as in those of the households. A presentation of the distribution of school provision is followed by an analysis of school access inequalities based on in-depth interviews conducted in eight districts of the suburbs of Oran.
\end{abstract}

Keywords: education supply - access to school - poverty - unplanned settlements - distance - public transport - qualitative analysis - Oran - Algeria.

\footnotetext{
* Enseignante-Chercheure au département d'Architecture et d'Urbanisme de l'Université des Sciences et de la Technologie d'Oran.

*** Chargé de recherche du ministère de l'Écologie, du Développement Durable, des Transports et du Logement (MEEDDTL) au LET, UMR CNRS 5593 - Université de Lyon (ENTPE, Université Lyon 2).
} 


\section{Introduction}

Rendre effectif l'accès à l'éducation pour tous représente un objectif majeur pour les pays du Sud. Nombre de travaux se sont concentrés sur l'importance de l'éducation dans les stratégies de réduction de la pauvreté, les relations entre éducation et pauvreté apparaissant complexes [Henaff, Lange, Martin, 2009]. En dépit de progrès dans la période récente, l'accès à l'éducation reste loin d'être universel. Ainsi, en Algérie, les nets progrès accomplis ces dernières décennies permettent d'atteindre des taux de scolarisation très élevés au primaire [Banque mondiale, 2007], même si la situation demeure moins favorable dans les zones rurales qu'en milieu urbain [Azzouz, 2003 ; Boulfekhar, Bedrouni, 2007]. Pourtant, à l'échelle de villes en forte croissance démographique et spatiale, les disparités géographiques dans l'offre éducative aux différents niveaux scolaires posent la question des inégalités sociospatiales d'accès à la formation et de réussite scolaire.

La question de l'accès à l'école dans les périphéries urbaines n'est ni récente, ni spécifique aux villes algériennes. L'inadaptation de l'offre scolaire, en quantité et en qualité, face aux besoins des enfants résidant dans les quartiers en urbanisation rapide, a été identifiée dès la fin des années 1980, à partir d'observations concernant les villes latino-américaines [Chauliac, 1989]. Les travaux portant sur les inégalités d'accès à l'école dans les villes africaines demeurent relativement peu nombreux, et portent principalement sur la partie subsaharienne du continent. Ces études aboutissent à des conclusions assez variables quant aux effets de la localisation de l'offre éducative sur la scolarisation des enfants en primaire. On observe notamment, selon les villes, une absence d'effet de la distance à parcourir sur la scolarisation à Accra [Canagarajah, Coulombe, 1998], un effet de la distance sur la non-scolarisation à Bamako limité aux filles [Zoungrana, Tokindang, Marcoux, 1998 et Konate, 1998], et un impact du sous-équipement des zones périphériques en établissements scolaires sur la faible fréquentation dans ces quartiers à Ouagadougou [Kaboré, Pilon, 2003] et à Bamako, mais comme facteur secondaire [Kaboré, Pilon, 2003]. À Conakry, la distance et la configuration spatiale des écoles publiques et privées ont un effet significatif sur la fréquentation du primaire [Diaz Olvera, Plat, Pochet, 2010]. Enfin, à Douala, comme à Conakry, les problèmes liés à la distance ou au manque de transport sont fréquemment mentionnés par les ménages, mais nettement moins souvent que la qualité de service de l'école publique, et le coût des établissements privés [Sitrass, 2004]. De façon globale, dans le contexte des villes subsahariennes, le coût de l'éducation est perçu comme la première difficulté limitant l'accessibilité à l'école, bien avant les problèmes liés à la qualité de l'enseignement et à l'inadéquation des prestations en regard des besoins, et les difficultés liées à la distance [Sikirou, 2008 ; Diaz Olvera, Plat, Pochet, 2010].

À notre connaissance, il n'existe pas d'études de ce type pour les villes algériennes. Cet article souhaite apporter un éclairage sur les contraintes et les difficultés rencontrées en matière d'éducation (aux niveaux primaire, moyen, et secondaire) des enfants des périphéries non loties d'Oran. Les périphéries oranaises sont tout à la fois marquées par une forte croissance démographique, une population jeune, un sous-équipement en services publics, notamment éducatifs et une desserte insuffisante par les transports collectifs. Elles sont également des lieux de concentration de ménages pauvres, fortement concernés par des difficultés à scolariser leurs enfants.

La question de l'accessibilité aux services urbains est multidimensionnelle, sociale et économique (coût d'accès aux services et dépenses induites), tout autant que de nature 
physique (distance, temps, efforts déployés, et moyens à disposition pour effectuer les déplacements) [Kaufmann, 2007]. La dimension sociale de l'accessibilité renvoie à de multiples facteurs : capital économique et culturel, réseau social mobilisable, compétences spatiales et connaissance de la ville acquises progressivement à partir des lieux, résidentiels ou non, fréquentés par les membres du ménage. Dans ce cadre, l'analyse va rechercher l'origine des difficultés rencontrées par les ménages, dans les facteurs qui relèvent de l'offre scolaire (dont les coûts de scolarisation, la qualité de l'enseignement, la localisation des établissements, la distance et les conditions d'accès à l'école au quotidien), comme dans les facteurs qui relèvent de la demande d'éducation et de ses déterminants (caractéristiques des ménages, dont les revenus, les problèmes de chômage et de santé, et les charges domestiques). Il s'intéressera également aux conséquences des difficultés de scolarisation pour les enfants concernés et pour leurs parents.

L'adéquation spatiale de l'offre scolaire à la demande par commune sera étudiée dans une première partie, à partir des statistiques de scolarisation, en distinguant les niveaux d'enseignement primaire, moyen et secondaire au sein de la wilaya ${ }^{1}$ d'Oran. Dans un second temps, les pratiques en matière de scolarisation des ménages pauvres résidant dans des communes périphériques d'Oran seront analysées à partir d'entretiens qualitatifs réalisés en fin d'années scolaires 2007 et 2008 [Rebouha, 2010].

\section{L'offre d'éducation à Oran}

\section{Le contexte de l'éducation en Algérie}

L'offre en éducation en Algérie est essentiellement publique, l'offre privée étant réduite à quelques établissements situés dans les grandes villes. Le système éducatif algérien comprend l'enseignement préparatoire, l'enseignement fondamental ${ }^{2}$ (qui regroupe le niveau primaire cinq ans et le niveau moyen - collège, quatre ans), l'enseignement secondaire, et l'enseignement supérieur.

L'offre s'est progressivement renforcée ces dernières décennies. En particulier, la création d'établissements dans les zones qui en étaient dépourvues a permis de démocratiser l'accès à l'éducation de base et de réduire les écarts de participation scolaire [Djebbar, 2008]. L'élaboration de la carte scolaire, qui repose sur les informations et données statistiques fournies par les wilayas et les communes concernant la population scolarisable et la fréquentation des établissements, a notamment pour objectif d'orienter les moyens affectés à l'éducation scolaire ${ }^{3}$. Ces dernières années, des efforts ont également visé à remédier au souséquipement des établissements (cantines, chauffage et autres aides ciblées). La forte croissance, sur longue période, de l'offre scolaire publique et gratuite ${ }^{4}$ a rendu possible

\footnotetext{
1. Collectivité publique territoriale, la wilaya constitue le premier niveau de division administrative en Algérie. La wilaya est subdivisée en dairas puis en communes (la wilaya d'Oran en compte respectivement 9 et 26). La commune est le niveau le plus fin pour lequel les statistiques sur l'éducation sont disponibles.

2. L'article 5 de l'Ordonnance du 16 avril 1976 portant organisation de l'éducation et de la formation rend l'enseignement obligatoire pour tous les enfants âgés de 6 à 16 ans révolus.

${ }^{3}$. Par ailleurs, la carte scolaire oblige à fréquenter l'établissement de sa zone mais les directeurs d'établissements peuvent accepter certaines dérogations, notamment du fait du manque d'offre. Des stratégies de contournement de la carte scolaire sont observées (utilisation de l'adresse d'autres membres de la famille pour inscrire les enfants dans des établissements à meilleure réputation).
}

4. L'éducation est gratuite en Algérie depuis 1962 ; les frais de scolarité sont symboliques. 
l'augmentation des taux de scolarisation en primaire et au collège. Cette croissance s'est maintenue au cours de la dernière décennie : entre 1999 et 2008, le taux de scolarisation des 6-15 ans est passé de $88 \%$ à $95 \%$ (Tableau 1). La poursuite de la réduction des écarts entre garçons et filles a permis d'atteindre une quasi-parité dans le primaire (98 filles pour 100 garçons en 2009, contre 96 filles pour 100 garçons en 1999) et dans le secondaire de premier niveau $^{5}$.

Toutefois, sur le plan des résultats, de nombreux problèmes demeurent, parmi lesquels des taux de réussite assez bas aux examens, des déperditions importantes entre niveaux (tableau 1) et des difficultés très fréquentes d'accès à l'emploi en sortie du système scolaire. Symptôme de ces difficultés qualitatives, les cours de soutien, privés et payants, connaissent un succès croissant à tous les paliers, du primaire à l'université. Les réformes engagées pour faire face à ces difficultés n'ont pas permis d'infléchir cette situation.

Tableau 1 - Indicateurs du système éducatif algérien de 1999 à 2008

\begin{tabular}{|c|c|c|c|c|c|c|c|}
\hline & \multicolumn{4}{|c|}{$\begin{array}{l}\text { Effectifs par niveau d'enseignement } \\
\text { (millions) }\end{array}$} & \multicolumn{2}{|c|}{ Résultats aux examens (\%) } & \multirow{2}{*}{$\begin{array}{c}\text { Taux de } \\
\text { scolarisation des } \\
6-15 \text { ans, }(\%)\end{array}$} \\
\hline & Primaire & $\begin{array}{l}\text { Moyen } \\
\text { (collège) }\end{array}$ & $\begin{array}{c}\text { Secondaire } \\
\text { (lycée) }\end{array}$ & Supérieur & $\begin{array}{l}\text { Brevet d'en- } \\
\text { seignement } \\
\text { fondamental }\end{array}$ & Baccalauréat & \\
\hline 1999-2000 & 4,8 & 1,9 & 0,9 & 0,4 & 44,0 & 28,7 & 88,5 \\
\hline $2000-2001$ & 4,7 & 2,0 & 1,0 & 0,5 & 41,0 & 33,0 & 90,6 \\
\hline $2001-2002$ & 4,7 & 2,1 & 1,5 & 0,5 & 37,0 & 30,0 & 92,9 \\
\hline $2002-2003$ & 4,7 & 2,2 & 1,1 & 0,6 & 34,0 & 30,0 & 93,0 \\
\hline 2003-2004 & 4,5 & 2,2 & 1,1 & 0,6 & 35,0 & 33,0 & 93,5 \\
\hline 2004-2005 & 4,3 & 2,2 & 1,1 & 0,7 & 41,6 & 30,6 & 93,6 \\
\hline $2005-2006$ & 4,1 & 2,2 & 1,1 & 0,7 & 60,3 & 44,6 & 93,7 \\
\hline $2006-2007$ & 4 & 2,4 & 1,0 & 0,8 & 43,9 & 43,7 & 94,8 \\
\hline $2007-2008$ & 3,9 & 2,5 & 0,9 & 0,9 & nd & nd & 95,4 \\
\hline
\end{tabular}

Source : ONS, Annuaires statistiques de l'Algérie $\mathrm{n}^{\circ} 21$ et $\mathrm{n}^{\circ} 25$ nd : non disponible.

Par ailleurs, cette croissance de l'offre d'éducation ne permet pas de combler totalement le déficit d'offre dans les zones périurbaines, ni d'éviter des situations de classes localement surchargées. Les situations décrites ci-après indiquent que ce déficit d'offre touche, à des degrés divers, les huit zones d'enquête, qu'elles soient non loties comme Med Boudiaf, Ain El Beida, ou Emir Khaled, ou qu'elles comprennent des parties loties, comme Hassi Ben Okba et Boufatis, quartiers qui ne comptent aucun établissement secondaire sur leur sol. En particulier, en l'absence d'établissement de proximité, les jeunes résidents scolarisés des bidonvilles d'Ain El Beida (quartier situé sur un oued et dont le cadre bâti est particulièrement précaire) sont acceptés par les écoles des quartiers limitrophes. Leur quartier n'étant pas équipé, les collégiens d'Hassi Ameur doivent se rendre à l'établissement de Hai Chahid Mahmoud, distant de $30 \mathrm{mn}$ à pied en moyenne. Hassian Ettoual, extension majoritairement lotie d'un noyau villageois, bénéficie de la présence d'écoles primaires (les deux écoles sont

\footnotetext{
${ }^{5}$. Source : Institut des statistiques de l’Unesco http://stats.uis.unesco.org/.
} 
situées à l'entrée et à la sortie du village, d'où des distances de marche non négligeables pour une partie des enfants scolarisés), et d'un collège qui accueille également les élèves de Med Boudiaf qui n'en est pas pourvu. Enfin, les lycéens de ces deux quartiers, tout comme ceux de Hassi Ben Okba et de Boufatis sont scolarisés au lycée de Ben Freha. Résidant loin de cet établissement, les lycéens en provenance de ces communes, notamment de Boufatis (commune située à $25 \mathrm{~km}$ du centre d'Oran) n'ont d'autre choix que de prendre les transports collectifs pour s'y rendre.

\section{La répartition de l'offre scolaire dans la wilaya d'Oran}

Au delà de ces quelques exemples, l'étude plus systématique de la répartition spatiale de l'offre d'éducation entre les différentes communes de la wilaya d'Oran utilise des données statistiques fournies par la direction de la Planification et de l'aménagement de la wilaya d'Oran (DPAT) et collectées par ses services auprès de la direction de l'Éducation d'Oran au niveau communal. Les établissements sont essentiellement publics et les quelques établissements privés situés dans la ville d'Oran n'apparaissent pas dans ces statistiques. Les différents indicateurs utilisés sont le nombre moyen d'élèves par classe à chaque niveau, le taux brut de scolarisation ${ }^{6}$ par niveau d'enseignement, et le nombre d'élèves ayant l'âge officiel de fréquenter un niveau d'éducation donné, rapporté au nombre de classes à ce niveau. Ces indicateurs renseignent sur la prise en charge de la demande potentielle, mais n'éclairent pas tous les aspects quantitatifs et qualitatifs de l'offre éducative.

Le niveau de précision géographique des données disponibles n'est pas suffisant pour faire apparaître les disparités spatiales qui pourraient exister entre les quartiers d'une même commune, par exemple entre quartiers lotis et quartiers d'urbanisation "spontanée ». Ce niveau de précision est toutefois suffisant pour représenter, à l'aide de cartes, les grandes tendances de répartition de l'offre dans l'espace urbain, et faire apparaître des écarts entre communes. La décomposition au niveau communal permet en particulier de mettre en évidence des différences dans l'offre et la fréquentation scolaires entre Oran, la communecentre, où résidaient en 2008 environ 609000 habitants, et les communes périphériques, dont la population était de l'ordre de 844000 habitants ${ }^{7}$.

Au niveau primaire, la répartition du nombre d'élèves par classe fait apparaître deux groupes de communes (Carte 1) : le premier se situe autour de 30 élèves par classe, à l'image de la commune d'Oran. Un taux d'occupation de plus de 35 élèves par classe est caractéristique du second groupe, qui représente plus de la moitié des communes périphériques (14). Avec près de 40 élèves par classe (de 37 à Hassi Ben Okba à 41 à Es Senia), les cinq communes où ont été menés les entretiens se situent toutes dans ce second groupe.

Au niveau du collège, le taux d'occupation moyen des classes est globalement élevé, entre 40 et 45 élèves par classe. Trois catégories de communes émergent. La catégorie plus importante numériquement (Oran et treize communes périphériques) assure l'enseignement des collégiens avec environ 40 élèves par classe (Carte 2). Cinq communes ont entre 45 et 55 élèves par classe. Enfin, cinq communes connaissent des surcharges à plus de 50 élèves par classe dont trois comptent 60 élèves ou plus par classe (jusqu'à 67 pour l'une des communes d'enquête, Hassi Ben Okba). Le nombre d'élèves par classe est également élevé à

6. Total des inscriptions dans un niveau spécifique d'éducation, sans distinction d'âge, exprimé en pourcentage de la population officiellement scolarisable au même niveau pour une année scolaire donnée, selon la définition de l'Unesco.

7. Selon les données de l'Office national des statistiques (ONS) provenant du recensement de population de 2008 sur la wilaya d'Oran. 
Es Senia (48), mais il se situe dans la moyenne de la wilaya d'Oran pour les trois autres communes d'enquête, avec moins de 40.

Carte 1 - Nombre moyen d'élèves par classe pour le niveau primaire en 2006, par commune, dans la wilaya d'Oran

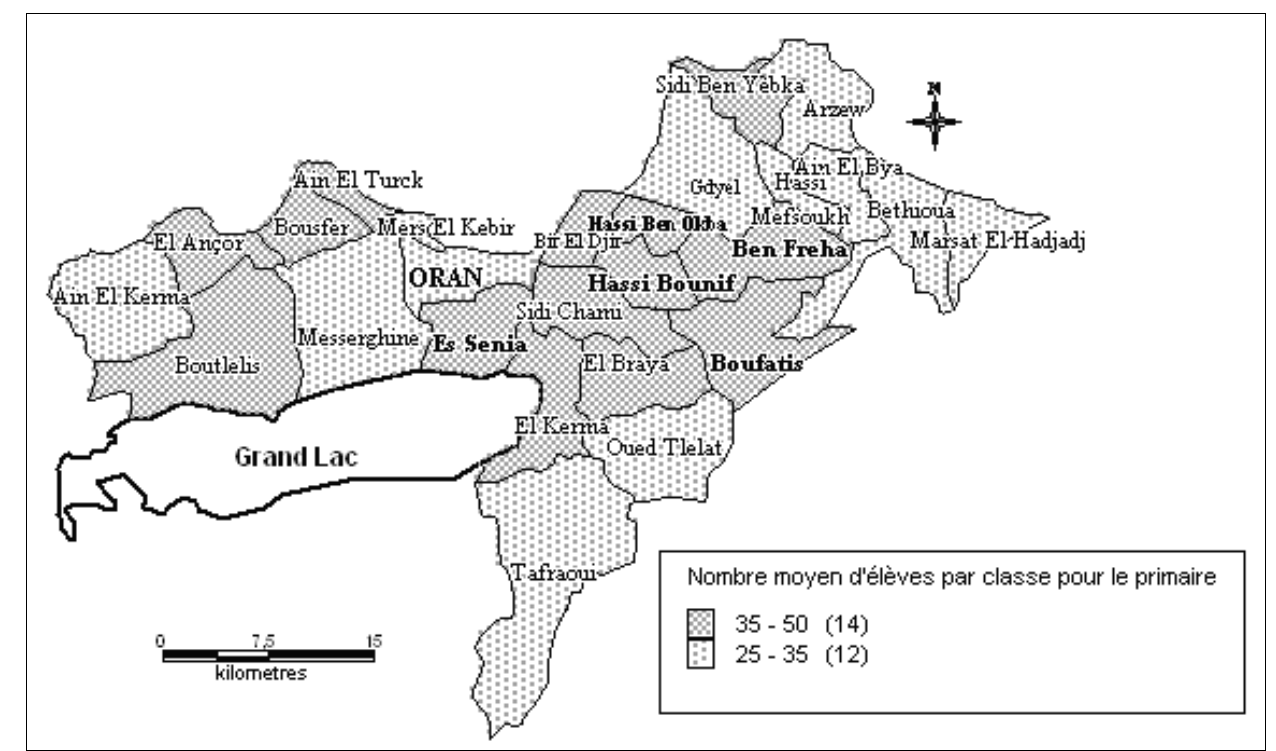

Source: Carte élaborée par les auteurs à partir des données de la direction de la Planification et de l'aménagement du territoire de la wilaya d'Oran [DPAT, 2007]

Les données de l'ONS issues du recensement de la population de 2008 fournissent le taux de scolarisation de la classe d'âge des 6-15 ans par commune (Tableau 2). Pour l'ensemble de la wilaya, environ $92 \%$ de la population scolarisable des 6-15 ans l'est effectivement. Dans les communes enquêtées, le taux est plus faible de quelques points par rapport à la ville-centre, et assez proche d'autres situations observées en périphérie.

Tableau 2 - Taux de scolarisation des 6-15 ans dans les communes enquêtées

\begin{tabular}{l|c|c|c}
\hline \multirow{2}{*}{ Communes } & \multicolumn{3}{|c}{ Taux de scolarisation des 6-15 ans } \\
\cline { 2 - 4 } & Garçons & Filles & Total \\
\hline Hassi Bounif & 89,4 & 89,3 & 89,3 \\
Es Senia (Ain el Beida) & 89,7 & 91 & 90,4 \\
Boufatis & 90,1 & 89,1 & 89,6 \\
Ben Freha (Hassian Ettoual) & 88,1 & 88,4 & 88,2 \\
Hassi Ben Okba & 89,7 & 89,6 & 89,6 \\
\hline Oran (ville) & 93,1 & 93,8 & 93,5 \\
Oran (ensemble de la wilaya) & 91,6 & 92 & 91,8 \\
\hline
\end{tabular}

Source : ONS, 2009.

Au niveau du lycée, les effectifs moyens par classe retrouvent des niveaux à priori plus satisfaisants : 25 élèves par classe à Oran, et moins de 30 élèves par classe dans neuf communes de périphérie, la situation apparaissant plus tendue dans des communes de périphérie proche, comme Hassi Ben Okba et surtout Es Senia et Messerghine (respectivement 33 et 44 élèves par classe). Mais si les effectifs par classe sont plus faibles au lycée qu'au collège, c'est en raison du taux très élevé d'abandons entre les deux cycles, le taux brut de scolarisation en lycée chutant à $33 \%$ en 2006. Les effectifs potentiellement 
scolarisables par classe se situent entre 105 et 170 élèves dans certaines communes limitrophes d'Oran, telles que Bir El Djir, Es Senia, Hassi Bounif, Sidi Chami. Seules les communes d'Ain El Turck avec un taux de 45 et de Bethioua avec 40 se distinguent par une offre nettement supérieure. Cette «surcharge potentielle» traduit le fait que les onze communes où les lycées sont absents envoient certains de leurs jeunes résidents étudier dans les communes limitrophes (Carte 3). Dans les communes enquêtées qui ne disposent pas de lycée, le taux de scolarisation à ce niveau se situe entre 13 et $20 \%$, en particulier à Hassi Ben Okba et Boufatis (à peine un enfant sur sept est scolarisé au niveau lycée), et à Hassi Bounif (à peine un sur cinq). Es Senia, commune équipée de lycée, se distingue par un taux brut de scolarisation nettement supérieur à ce niveau (plus de deux sur cinq).

Carte 2 - Nombre moyen d'élèves par classe au collège en 2006, par commune, dans la wilaya d'Oran

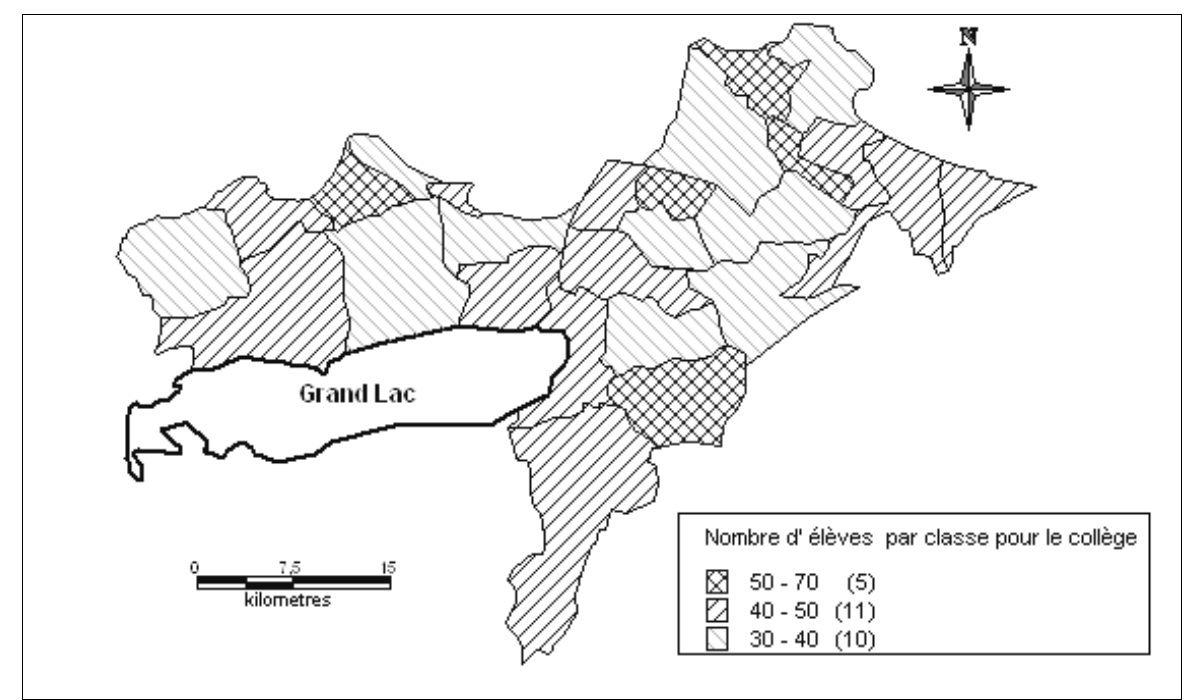

Source : Carte élaborée par les auteurs à partir des données de la DPAT, 2007

Carte 3 - Effectifs potentiellement scolarisables par classe au lycée, par commune, dans la wilaya d'Oran en 2006

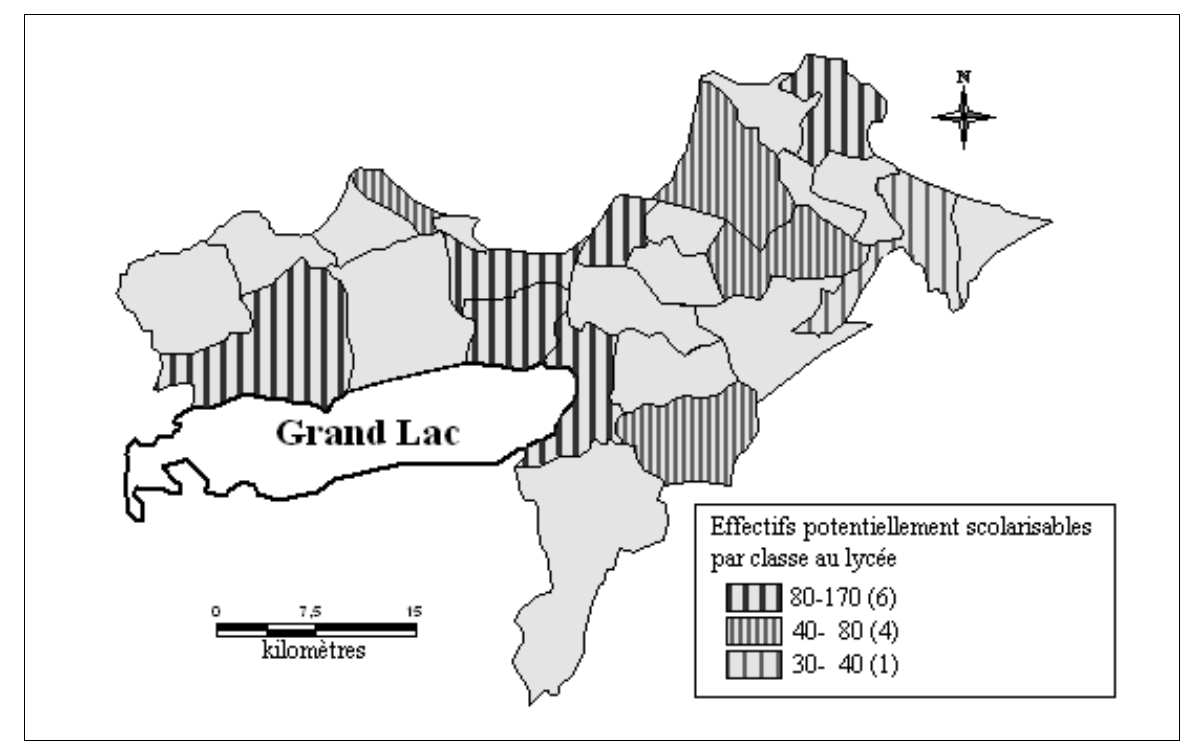

Source : Carte élaborée par les auteurs à partir des données de la DPAT, 2007. Les communes non hachurées ne disposent pas de lycée sur leur territoire. 
De façon générale, le taux d'occupation élevé des classes dans certaines communes met en évidence des conditions d'études rendues plus compliquées par une offre insuffisante. Les retards pris par rapport aux objectifs de construction d'infrastructures scolaires [Barti, 2009] contribuent au problème assez général de surcharge des classes que connaissent les cycles primaire et moyen. Le sous-équipement en établissements scolaires se manifeste de façon plus ou moins aiguë selon les communes, les communes périphériques étant généralement moins bien fournies en services d'éducation que la commune centre, en particulier celles qui ont connu une urbanisation plus rapide et une forte croissance de la population. Le déficit apparaît dès le niveau moyen et s'amplifie pour le secondaire

\section{Les raisons d'une scolarisation contrariée}

L'analyse des inégalités dans l'accès à l'école est basée sur des entretiens menés en 2007 et 2008 auprès de soixante ménages, dans huit quartiers de la périphérie d'Oran. Dans la mesure du possible (possibilité de rencontrer les personnes à leur domicile, accord de chacun, âge), tous les membres du ménage, adultes et enfants, ont été interrogés individuellement, de façon à recueillir les différents points de vue sur une situation en partie commune. Ces entretiens n'ont pas pour vocation de représenter la situation des familles résidant en périphérie d'Oran, mais de mettre en évidence la diversité des situations de pauvreté, et leurs conséquences sur l'accès aux équipements et services collectifs, notamment à l'école.

\section{Une approche qualitative des difficultés d'accès à l'éducation}

Les huit quartiers dans lesquels a été menée l'enquête font partie de cinq communes périphériques : Es Senia, Hassi Ben Okba, Hassi Bounif, Ben Freha, et Boufatis (Carte 4). Ces quartiers ont été choisis pour refléter des situations diverses en termes de distance au centre d'Oran, de desserte en transports en commun, d'équipements, d'emploi local, et de niveau de vie des populations résidantes. Les ménages enquêtés sont d'origine géographique variée et n'ont pas tous le même niveau économique ou d'éducation, même si les parents sont fréquemment de niveau primaire ou sans instruction. Ainsi, par exemple, près du tiers des adultes de Hassi Bounif, Boufatis et Hassi Ben Okba sont sans instruction.

Évaluer le degré de pauvreté des ménages enquêtés n'est pas aisé, car dans certains ménages, tous les revenus n'ont pas été déclarés, la taille des ménages très variable (d'une à douze personnes) ne facilitant pas les comparaisons. Toutefois, la grande majorité des ménages rencontrés peuvent être classés parmi les bas revenus. La distribution s'établit comme suit, pour les ménages dont les différents membres ont communiqué leur revenu, un point de comparaison étant le salaire minimum, fixé à $12000 \mathrm{DZD}^{8}$, au moment de l'enquête : la moitié des ménages a de très faibles revenus, depuis ceux déclarant n'avoir aucun revenu à ceux dont les revenus cumulés, généralement issus de petits métiers de l'informel n'excèdent pas 10000 DZD mensuels. Ces revenus sont de plus assez instables d'un mois sur l'autre ; un ménage sur trois dispose de 10000 à 20000 DZD par mois : ces revenus proviennent alors souvent du cumul des revenus de plusieurs actifs de l'informel, de petits fonctionnaires ou d'employés ; enfin, un ménage sur six a un revenu un peu supérieur, situé dans une fourchette allant de 20000 à 40000 DZD.

8. Soit $120 €$ mensuels $; 100$ dinars $(D Z D)=1$ euro 
Carte 4 - Communes des terrains d'enquête dans la wilaya d'Oran

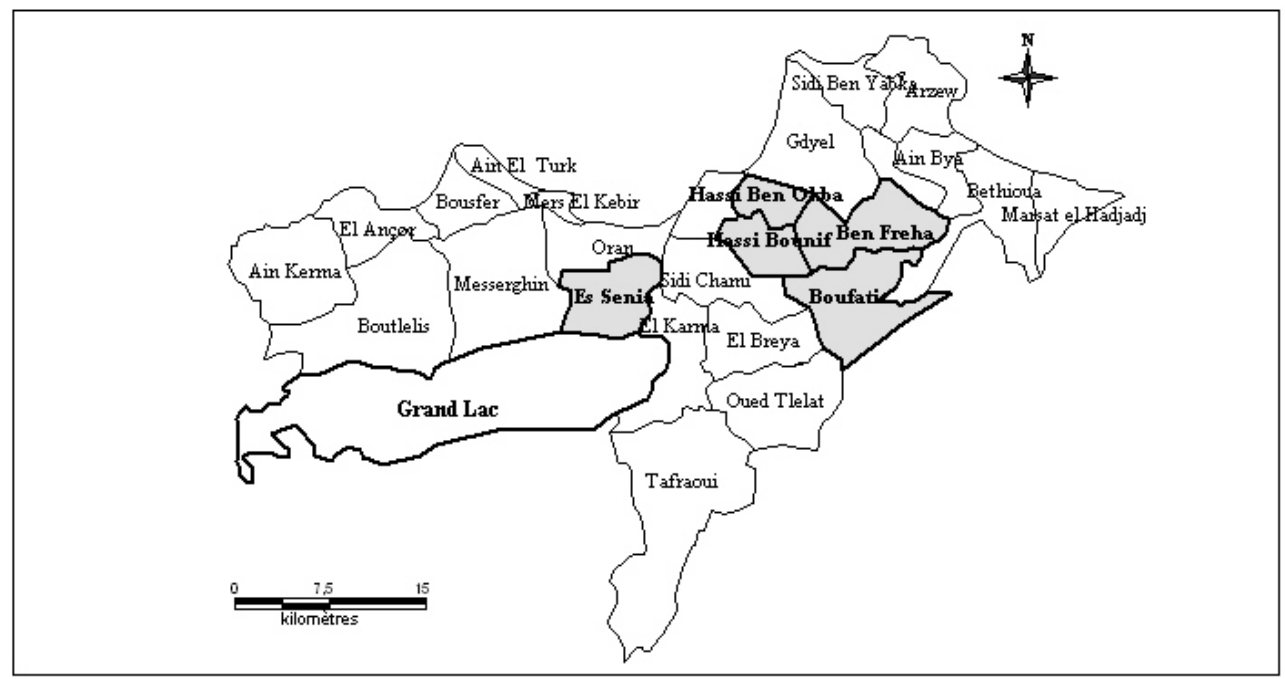

Les entretiens ont notamment abordé les thématiques liées à l'habitat et aux changements de résidence, les questions d'accès aux activités dans la ville, et la mobilité quotidienne à la fois par le recueil des pratiques (déclarées) et des appréciations et opinions vis-à-vis du quartier de résidence, de sa desserte en transport public, et de l'accès aux activités [Rebouha, 2010]. Nombre de foyers interrogés étaient concernés par les thèmes de l'école, les causes de l'échec scolaire, l'avenir et les attentes des jeunes, les dépenses scolaires, les stratégies et les contraintes de scolarisation. L'analyse des entretiens a eu pour objectif de repérer les éléments défavorables à la scolarisation des enfants et de chercher à comprendre s'ils avaient un lien avec le fait d'habiter dans une zone périphérique relativement sous-équipée et éloignée. Ces entretiens ont été effectués en 2007 et 2008, à la fin du troisième trimestre, pendant la période de vacances scolaires, pour prendre en compte les difficultés scolaires vécues au cours de l'année écoulée (parcours scolaire, niveau scolaire, réussite, redoublement...) ${ }^{9}$.

\section{La pauvreté, un élément déterminant pour la scolarisation}

Une grande partie des ménages rencontrés est logée dans des habitations vétustes et trop petites [Rebouha, 2010]. Dans un domicile exigu, les enfants ne disposent pas des moyens élémentaires pour leurs activités scolaires (espace de travail, table, lampe...). Par ailleurs, les habitations non raccordées au réseau d'eau obligent les enfants à se charger de l'approvisionnement : "Je ramène l'eau tous les jours, c'est aussi moi qui ramène la bouteille de gaz butane » (Hassi Ben Okba, garçon de 13 ans). À partir des entretiens, il n'est pas possible d'établir un lien direct entre l'insalubrité assez fréquente des conditions de logement (problèmes d'écoulement des eaux usées, stagnation d'ordures), les problèmes de nutrition, l'état de santé des enfants, et la scolarisation. La littérature scientifique montre cependant que ce lien existe, même s'il y a des débats sur l'impact du choix de la mesure sur les résultats obtenus [Handa, Peterman, 2007]. Les problèmes de santé des enfants ne sont pas rares dans les familles rencontrées, et peuvent entraîner la déscolarisation : «Ma fille de 11 ans [...], j'ai essayé de la ramener plusieurs fois à l'école, mais elle refuse, elle ne peut plus suivre ses études. Elle est malade, l'école c'est loin, et elle oublie vite ses leçons [...]. Pour consulter un médecin, on n'a pas les moyens pour payer » (Ain El Beida, femme de 34 ans). L'état de

\footnotetext{
${ }^{9}$. Les entretiens ont été menés en arabe, puis retranscrits en français par F. Rebouha.
} 
santé précaire de l'un des parents, en fragilisant sa situation par rapport à l'emploi, peut également entraîner de grandes difficultés de scolarisation des enfants.

Le coût de l'éducation pour les ménages englobe, outre des frais de scolarité relativement abordables $^{10}$, des cotisations diverses, des frais de fournitures scolaires, les livres scolaires, d'habillement, les éventuels frais de transport quand l'établissement est éloigné du domicile et les éventuels cours particuliers et de soutien scolaire... Bien que des aides (fournitures scolaires, prêt de livres) puissent être attribuées aux ménages en difficulté financière par la direction des établissements scolaires, le financement de la scolarisation des enfants est souvent très difficile chez les ménages à bas revenus. L'accumulation des coûts de scolarisation rend parfois impossible leur prise en charge par les familles lorsque les budgets sont très serrés, comme dans le cas de ce ménage où une mère de quatre enfants qui travaille et a confié la garde du foyer à l'aînée de ses filles de 18 ans, déscolarisée, déclare : «Comment je vais faire pour lui [son jeune fils] acheter un stylo ? J'ai ma fille qui est scolarisée à Bounif, avec qui je ne m'en sors pas, tous les jours elle a besoin de 20 dinars [...] des fois j'emprunte, mon fils de 20 ans a arrêté l'école à cause du transport, à cause de 20 dinars, il marchait à pied [pour se rendre au lycée], alors qu'il était très bon élève » (Hassi Ameur, femme de 42 ans).

Des aides existent pour l'achat des fournitures scolaires en début d'année, mais aux dires de nombre de ménages interrogés, elles semblent insuffisantes et mal réparties. Les ménages en situation de grande pauvreté ne bénéficient ni d'une aide sociale spécifique, ni d'un accompagnement tout au long de l'année. Face aux dépenses importantes comme l'achat des fournitures scolaires et les frais d'inscription, les ménages essaient de s'en sortir avec l'aide de leur voisinage ou de leur famille, ou en recourant au crédit : "On a beaucoup de dépenses pour la fourniture scolaire, on a jusqu'à 10000 dinars, des fois on emprunte. Ma fille, les livres, les cahiers, ça lui fait 2000 dinars, et on a payé 2000 dinars pour qu'elle passe son diplôme de BEF » (Emir Khaled, femme de 40 ans). L'autre alternative consiste à faire des restrictions sur les dépenses, et de ne se procurer que les livres des matières paraissant les plus indispensables. Dans ce cas de figure, les dépenses de scolarisation peuvent entraîner des redoublements, un décrochage, ou l'abandon: "J'ai fait arrêter l'école à ma pauvre fille de 15 ans, elle était en $7^{\mathrm{e}}$ année [collège], parce qu'on n'a pas les moyens d'acheter les fournitures scolaires » (Med Boudiaf, femme de 43 ans).

Les difficultés qu'éprouvent les enfants à assumer les signes extérieurs de pauvreté peuvent également contribuer à une démobilisation de leur part : "J'ai mon fils de 14 ans qui aime s'habiller, et parce que mon mari et moi n'avons pas les moyens de lui acheter de beaux habits, il a honte de sortir dans la rue et même pour les études, il ne veut pas aller au lycée [...]. Il se sent différent » (Hassi Ameur, femme de 37 ans).

Les entretiens réalisés montrent que la scolarisation des plus grands s'arrête lorsqu'il faut assumer financièrement celle des plus jeunes enfants. Les frais trop élevés nécessités par la scolarisation sont la raison la plus fréquemment évoquée pour expliquer les abandons scolaires et les difficultés rencontrées par les enfants pour suivre en classe, les problèmes pour se rendre à l'établissement du fait de son éloignement étant également mis en avant.

\footnotetext{
${ }^{10}$. Ces frais de scolarité comprennent les frais d'inscription annuels, de l'ordre de 50 DZD pour le primaire, comprenant le cahier scolaire et la cantine quand celle-ci existe (un développement des cantines est observable depuis l'année 2008-2009), entre 150 et 75 DZD pour l'enseignement moyen, et entre 200 et 100 DZD pour le lycée selon l'année, et le cas échéant, les frais d'inscription aux examens du BEF (brevet d'enseignement fondamental) et du baccalauréat.
} 


\section{L'environnement familial, facteur de réussite ou d'échec scolaire}

«L'environnement social de l'enfant est un élément capital pour l'envoi et le maintien des enfants à l'école » [Konate, Guèye, Neska Vita, 2003, p. 17]. Le constat établi pour la scolarisation au Mali vaut pour les situations analysées à Oran à partir du corpus d'entretiens, d'où trois types d'environnements familiaux se distinguent :

- Des parents investis dans la scolarité de leurs enfants, qui représentent une minorité des adultes rencontrés. Le niveau de vie et le niveau d'instruction du ou des parents apparaissent déterminants dans leur engagement dans le parcours scolaire de leurs enfants, le coût financier mais aussi temporel de cet investissement, vraisemblablement moins important lorsque les revenus ou le niveau d'éducation des parents sont plus élevés [Caillaud, 2004], pouvant représenter un élément d'explication. Les parents dont les contraintes financières ne sont pas trop fortes se mobilisent pour la réussite scolaire et font donner à leurs enfants des cours de soutien dans une matière ou deux selon leurs moyens, contribuant à l'essor de ces cours [C.L., 2005 ; N.L., 2007]. Une jeune lycéenne explique ainsi : «Je ne fais que les maths [en cours particulier]. Deux heures par semaine, c'est le lundi après-midi. Ça coûte 1000 dinars par mois. Je vais à Oran, parce qu'ici, il y en a mais ils n'enseignent pas bien, donc je préfère aller à Oran » (Boufatis, jeune fille au lycée de Ben Freha, 16 ans).

- Des parents désinvestis qui négligent l'intérêt de s'impliquer dans la scolarité de leurs enfants. En particulier, l'absence d'investissement du chef de famille dans la scolarisation des enfants peut favoriser la non-scolarisation ou l'interruption du parcours scolaire. Ces cas de figures, que nous n'avons que rarement rencontrés au cours de nos entretiens, existent néanmoins, témoin cet extrait où le désinvestissement apparaît général : « Ma sœur de 16 ans n'a jamais été à l'école [...] Elle n'a pas fait ses vaccins [...] et mon père ne s'est pas occupé d'elle, il n'a pas cherché à faire les démarches nécessaires, alors elle a été délaissée, elle n'a pas pu être à l'école » (Hassi Bounif, jeune fille de 19 ans). Il reste à évaluer les éléments explicatifs de tels désengagements. D'autres abandons, plus soudains, peuvent intervenir suite à un divorce, comme le raconte cette femme " Il [le père] s'est remarié et il nous a expulsés de la maison. Après le divorce, il n'y avait personne pour leur acheter la fourniture scolaire. Tous mes enfants ont quitté l'école » (Ain El Beida, femme de 59 ans).

- Des parents contraints de scolariser leurs enfants dans de mauvaises conditions, ou de ne pouvoir assumer la poursuite de leur scolarisation. Ce sont de loin les plus nombreux des cas que nous avons rencontrés, notamment parmi les familles dont les revenus sont très bas.

Toutefois, les caractéristiques des ménages, la faiblesse des revenus, un climat familial peu propice, la précarité des conditions de logement ne sont pas les seuls facteurs d'échec scolaire. L'analyse des entretiens pointe également l'influence d'un environnement scolaire inadapté aux situations des foyers pauvres de périphérie comme un facteur pertinent dans la réussite scolaire.

\section{Un environnement scolaire inadapté aux milieux défavorisés}

Dans les quartiers, généralement non lotis, de périphérie, la concentration importante d'élèves en difficulté d'une part, et le déficit de services d'éducation, au niveau secondaire notamment, sont loin de permettre l'équité des conditions de scolarisation, alors que la réussite scolaire y exigerait la prise en charge des cas les plus complexes. Dans une étude consacrée à l'échec scolaire en Algérie, et prenant comme critère l'avis des instituteurs, Maache et Seghir Chorfi 
mettent en évidence le rôle des modes de fonctionnement de l'école dans l'échec et le faible rendement scolaires [2002]. A contrario, la qualité de l'accueil et la mise en place de services spécialisés peuvent permettre une prise en charge individualisée : «Moi, j'étais chez un psychologue à Gdyel, j'étais malade, j'avais des troubles psychologiques. Maintenant je vais bien. Au lycée, c'est le directeur qui m'y a emmené [...]; j'avais de fréquentes crises de colère. Je suis resté quatre mois chez le psychologue, puis j'ai arrêté » (Med Boudiaf, garçon de 21 ans, en $1^{\text {re }}$ année universitaire). Pour autant, dans les entretiens, ce n'est pas le manque de moyens des établissements scolaires, les classes surchargées ou les conditions de travail difficiles du corps enseignant qui sont mises en cause, mais le comportement ou les compétences des enseignants, à l'image de cet extrait : "J'ai un fils, il a 15 ans, il a arrêté en $6^{\mathrm{e}}$ année. Ça ne lui a pas plu, les études, il m'a dit : "on ne nous explique pas la leçon ni rien du tout", alors il en avait assez [...]. Ma fille m'a dit qu'“ils ne nous expliquent pas et ils nous crient dessus" ; ils leur font peur et ils deviennent frustrés et craintifs » (Hassi Bounif, femme de 51 ans).

Le manque de moyens des établissements ne leur permet pas d'accueillir les élèves résidant dans des quartiers éloignés dans de bonnes conditions. Aussi, une partie de ces lycéens, dans l'impossibilité de rentrer chez eux, passent la pause de midi à l'extérieur de l'établissement : «Nous restons dehors, à l'extérieur [entre 12 heures et 14 heures] et nous achetons des sandwichs à 5 ou 10 dinars ou bien nous ramenons avec nous. Ça nous revient à 50 dinars la journée. [...] Nous nous asseyons sur le trottoir ou nous passons notre temps à marcher» (Hassi Ben Okba, fille 16 ans au lycée). À nouveau, les insuffisances de l'offre éducative (éloignement, absence de cantine) apparaissent plus pénalisantes pour les enfants des quartiers périphériques, et ce, d'autant plus que les conditions de transport sont généralement éprouvantes.

\section{Déplacements quotidiens : vicissitudes liées à la disponibilité de l'offre de transport}

Selon une enquête sur les transports menée dans l'agglomération oranaise, l'éducation est le premier motif de déplacement dans la ville d'Oran et ses périphéries (43\% du total), avec une prédominance de la marche à pied qui constitue les deux tiers des déplacements [Betur, 2000]. Les résultats publiés de cette enquête ne rendent cependant pas compte des contraintes et conditions de déplacements des jeunes scolarisés: déplacements pédestres se pratiquant souvent sur des chemins escarpés et caillouteux, parfois jonchés de déchets, notamment dans les quartiers non lotis... Il est important de rappeler la grande variabilité des conditions de déplacement : au sein d'un même quartier, les résidents n'ont pas les mêmes caractéristiques de trajet, ni les mêmes contraintes. L'école primaire installée en début de quartier va se situer de plus en plus loin des habitants des parcelles les plus récemment urbanisées. Dans l'exemple suivant, l'éloignement de l'école exige une durée de marche quotidienne très élevée, de l'ordre d'une heure et demie, compte tenu des déplacements domicile - école de la mi-journée : "Mon petit garçon de 10 ans met vingt à vingt-cinq minutes pour y arriver et il refait les années [il redouble]. Quand ils lui ont changé d'école, il a été très perturbé » (Hassian Ettoual, femme de 38 ans).

Toutefois, même dans les quartiers informels comme les douars, la population bénéficie généralement de la construction d'écoles primaires; en revanche collèges et lycées sont rarement prévus, aussi est-ce à partir du collège que les conditions d'accès se compliquent au 
quotidien, d'autant que le transport scolaire est très restreint dans la wilaya d'Oran (26 opérateurs exploitant 35 véhicules ${ }^{11}$ pour 26 communes).

Les disparités en matière de distribution des établissements scolaires, notamment les collèges et plus encore les lycées, font que les élèves résidant dans les zones périphériques enquêtées dépendent dans la plupart des cas de l'offre de transport collectif artisanal pour joindre les collèges situés dans les quartiers ou communes limitrophes. Or les arrêts sont souvent distants du domicile ou de l'établissement, imposant alors de longs trajets terminaux à pied. En outre, les élèves partagent les transports en commun du secteur artisanal ou les bus avec d'autres usagers : ayant moins d'expérience de l'usage du transport en commun que les adultes, considérés comme non prioritaires par les exploitants et stigmatisés par l'incivilité de certains, ils rencontrent énormément de problèmes dans ces déplacements. À l'instar d'autres villes du Maghreb comme Tunis ou Casablanca [Godard, 2008], les scolaires du niveau fondamental (mais pas les lycéens), bénéficient de demi-tarifs, ce qui en fait une clientèle moins lucrative aux yeux des transporteurs et contribue à leur refoulement aux heures de pointe. Les témoignages sont très nombreux, dénonçant la rareté des véhicules et le refus des opérateurs de transport de faire monter les élèves aux heures de pointe. Le fait de renoncer à monter dans les véhicules et d'effectuer une longue distance de marche entraîne des retards aux cours. «Je préfère marcher plutôt que de prendre le bus, le bus ne vient pas tôt et on ne nous fait pas monter, parce que les élèves créent l'anarchie [...]. On attend tous dans le même arrêt, quand les transporteurs arrivent, ils refusent de nous faire monter » (Hassi Ameur, collégienne de 14 ans). «Parfois, on fait le trajet à pied, c'est parce que quelques élèves ne payent pas leur place » (Hassi Ameur, collégienne de 15 ans).

Les disparités de répartition des lycées et leur nombre limité au sein de la wilaya d'Oran en rendent l'accès particulièrement pénible pour les lycéens : «Il faut que je me lève tôt et tout, je vais prendre le transport, souffrir comme souffrent tous les gens, les bousculades et tout, et encore, des fois, on ne trouve pas le transport, ce qui fait, tu es obligée d'aller à pied entre Ben Freha et Boufatis » (Boufatis, fille de 17 ans au lycée de Ben Freha). «Pour le transport, ça dépend, des jours nous trouvons facilement, mais parfois, on trouve dans un autre coin, puis dans autre coin on ne trouve pas, on attend beaucoup, une heure » (Hassi Ben Okba, fille de 16 ans, au lycée). En effet, les opérateurs de transport changent parfois de station, lorsque la demande est importante, pour éviter que leur véhicule ne soit endommagé. Ils préfèrent alors s'éloigner du terminus, et c'est aux usagers de repérer l'endroit où les transporteurs s'arrêtent.

Si les parents mettent l'accent sur le coût économique des déplacements et les difficultés de scolarisation liées à la distance entre le domicile et le lieu de formation, les adolescents scolarisés, quant à eux, ont tous un commentaire pour dépeindre les conditions dans lesquelles s'effectuent les trajets, le temps perdu, la pénibilité, et la fatigue qui en découlent. Dans tous les cas, une localisation en périphérie non lotie tend à se traduire par un cumul d'obstacles, entre les conditions de logement précaires, l'éloignement des établissements, et par conséquent les déplacements plus longs, plus difficiles et plus coûteux.

11. Chiffres fournis par la direction de la Planification et de l'aménagement du territoire (DPAT). 


\section{Conclusion}

Les obstacles à la scolarisation des enfants dans les périphéries faiblement urbanisées apparaissent à la fois nombreux et intriqués, reflets du caractère multidimensionnel de la pauvreté et de la complexité des relations entre pauvreté et éducation [Henaff, Lange, Martin, 2009]. L'étude du cas des périphéries d'Oran montre toute la difficulté qu'il y a, dans les faits, à analyser séparément les obstacles à la scolarisation, et encore plus à les hiérarchiser, tant les discours mettent en évidence qu'ils sont imbriqués et interagissent pour produire des situations ou des parcours de scolarisation globalement très problématiques. Le principal point commun en est le caractère déterminant de la faiblesse des revenus des populations résidantes, qui ne permet pas aux ménages de faire face aux exigences financières de la scolarisation. Le caractère peu adapté de l'offre d'éducation à des contextes d'apprentissage difficiles ressort également des entretiens. L'analyse de la répartition spatiale de l'offre d'établissements scolaires, comme les discours de parents et d'adolescents scolarisés laissent entrevoir que les enjeux de l'accessibilité physique aux lieux de formation sont loin d'être mineurs.

En conséquence de ces difficultés, l'école est de moins en moins vue comme un moyen d'accès à la vie active par les jeunes des quartiers défavorisés d'Oran, qui tendent à ne pas poursuivre leurs études. La sortie définitive du système scolaire se traduit pourtant, dans la majorité des cas, par une période longue de non-travail, et de fortes difficultés d'accès à l'emploi [Doumandji, Ziane, 2006], entre inactivité et désœuvrement ou « petits boulots », le plus souvent au quartier [Rebouha, Pochet, 2009]. L'arrivée à l'âge adulte de nouvelles populations non qualifiées dans les territoires périurbains contribue à renforcer les tensions sur le marché local de l'emploi, la précarisation des conditions de vie, et les inégalités dans les marges urbaines d'Oran. Si l'objectif d'un accroissement quantitatif de l'accès à l'école a été tenu en Algérie, tout au moins pour l'enseignement primaire, l'exemple des périphéries d'Oran montre que subsistent des inégalités sociales et spatiales structurantes en matière d'accès à l'éducation et à la formation. 


\section{Bibliographie}

Azzouz L. [2003], "Disparités géographiques et déroulement de la scolarité », Sciences Humaines, $\mathrm{n}^{\circ} 19$, Constantine, Université Mentouri, p. 45-60.

BANQUE MONDIALE [2007], «République Algérienne Démocratique et Populaire. À la recherche d'un investissement public de qualité : une revue des dépenses publiques », Rapport $n^{\circ} 36270-D Z$, Washington, Groupe pour le développement socio-économique, Région Moyen-Orient et Afrique du Nord, 199 p. :

http://siteresources.worldbank.org/INTALGERIAINFRENCH/Resources/ALGERIA.PER.French.V olumeI.pdf (page consultée le 29 octobre 2010).

BARTI H. [2009], «Retard dans le programme des infrastructures scolaires », $1^{\text {er }}$ juillet, Le quotidien d'Oran : http://www.lequotidien-oran.com/index.php?news $=5123279 \&$ archive_date $=2009-07-01$ (page consultée le 29 octobre 2010).

BELBACHIR N. [2010], «l'Algérie a consacré $5 \%$ du PIB pour le secteur de l'Éducation », La Tribune, 19 juin, p. 9 : http://www.latribune-online.com/pdf/2010/06/19062010.pdf (page consultée le 29 octobre 2010).

BENRAMDANE F. [2008], «Algérie - Rentrée scolaire 2008 : Comment et pourquoi va-t-elle dérégler le système éducatif (jusqu'à 2015) et universitaire (jusqu'à 2024) ? », El Watan, 4 octobre 2008, p. 23.

BETUR [2000], Enquête ménage Transport, Oran 2000, Rapport final, Alger, groupement EMA/BETUR-CNEAP.

BOULFEKHAR N., BEDROUNI M. [2007], «Les déterminants familiaux de la scolarisation en Algérie », $5^{e}$ Conférence sur la Population Africaine, Arusha, 10 au 14 décembre, 12 p.: http://uaps2007.princeton.edu/download.aspx?submissionId=70535 (page consultée le 29 octobre 2010).

C.L. [2005], « Soutien scolaire. Une dérive nommée "cours à domicile" », El Watan, 19 octobre, p 9.

CAILlaUd F. [2004], «Les inégalités sexuelles dans l'accès à l'éducation, quelles conséquences pour la politique éducative des pays en développement? », Journées scientifiques le financement du développement et réduction de la pauvreté, Marrakech, 23 p.: http://www.aed.auf.org/IMG/pdf/F.CAILLAUD.pdf (page consultée le 29 octobre 2010).

Canagarajah S., Coulombe H. [1998], « Child labor and schooling in Ghana », Policy Research Working Paper $\mathrm{n}^{\circ} 1844$, Washington D.C., World Bank, 44 p.: http://wwwwds.worldbank.org/servlet/WDSContentServer/WDSP/IB/1997/11/01/000009265_3980203115439/ Rendered/PDF/multi_page.pdf (page consultée le 2 avril 2011).

CHAULIAC M. [1989], «Éducation, santé et conditions de vie des enfants d'âge scolaire dans les zones à urbanisation rapide », in SALEM G., JEANNEE E. (dir.). Urbanisation et santé dans le Tiers Monde: transition épidémiologique, changement social et soins de santé primaires, Paris, ORSTOM, p. 395-400.

Diaz Olvera L., Plat D., Pochet P. [2010], «À l'écart de l'école ? Pauvreté, accessibilité et scolarisation à Conakry », Tiers Monde, n 202, p. 167-183.

DJEBBAR A. [2008], «Le système éducatif algérien : miroir d'une société en crise et en mutation », in ChenNTOuF T. (dir.), L'Algérie face à la mondialisation, Dakar, CODESRIA, p. 164-207.

DOUMANDJI G., ZIANE S. [2006], «Déscolarisation, pauvreté et travail des enfants et jeunes adolescents en Algérie », Sciences humaines, ${ }^{\circ}$ 25, p. 19-35, Constantine, Université Mentouri.

DPAT [2007], Annuaire Statistique de la wilaya d'Oran 2006, Oran.

GODARD X. [2008], « Obstacles à la mobilité durable dans les villes méditerranéennes, convergences et variété des contextes et trajectoires », Conférence CODATU XIII, Hô Chi Minh Ville, 12-14 nov., 10 p. : http://www.euromedina.org/bibliotheque_fichiers/Doc_TransportMobiliteDurable.pdf, (consulté le 29 octobre 2010). 
Handa S, Peterman A.[2007], «Child Health and School Enrollment: A Replication », Journal of Human Resources, vol. 42, $\mathrm{n}^{\circ}$ 4, p. 863-880.

Henaff N., LANGe M.-F., MARTin J.-Y. [2009], «Revisiter les relations entre pauvreté et éducation », Revue française de socio-économie, vol. 1, n 3, p. 187-194.

JOURNAL OFFICIEL DE LA REPUBLIQUE ALGERIENNE [2009], nº78, Loi n ${ }^{\circ}$ 09-09 du 30 décembre 2009 portant loi de finance pour 2010, 31 décembre.

JOURNAL OFFICIEL DE LA REPUBLIQUE ALGERIENNE [2010], n80, Loi n 10-13 du 29 décembre 2010 portant loi de finance pour 2011, 30 décembre.

KABORÉ I., PILON M. [2003], «Intra-urban enrolment disparities in Ouagadougou », Background paper prepared for the Education for All Global Monitoring Report 2003/4, Gender and Education for All : The Leap to Equality, 2004/ED/EFA/MRT/PI/31, Unesco, 16 p.

KAUFMANN V. [2007], «La motilité : une notion clé pour revisiter l'urbain ?», in BASSAND M., KAUFMANN V., JOYE D. (dir.), Enjeux de la sociologie urbaine, Lausanne, Presses polytechniques et universitaires romandes, p. 171-188.

KoBIANE J.-F. [2002], « De la campagne à la ville, constances et différences dans les déterminants de la scolarisation des enfants au Burkina Faso », Colloque La recherche face aux défis de l'éducation au Burkina Faso, Ouagadougou, INSS/CNRST-IRD-AREB, 19 au 22 novembre, 18 p.

KonATE M.K., GUEYE M., NESKA ViTA T. [2003], « Scolarisation des enfants au Mali selon le profil des ménages et étude de leur maintien à l'école », Background paper prepared for the Education for All Global Monitoring Report 2003/4, Gender and Education for All: The Leap to Equality, 2004/ED/EFA/MRT/PI/38, Unesco, 19 p.

MAACHE Y., SEGHIR CHORFI M. [2002], «L'enfant en situation d'échec scolaire face aux enseignants. Brefs regards sur des pratiques pédagogiques », Sauvegarde de l'enfance, vol. 57, n 4, p. 156-162.

N.L. [2007], «Cours particuliers, le temps de l'école parallèle et lucrative », 24 octobre, El Watan, p. 11.

ONS [2009], Données statistiques du recensement de 2008 de la wilaya d'Oran, ${ }^{\circ}$ 527/31, Alger, $28 \mathrm{p}$.

Rebouha F. [2007], "Concentration des services de santé, contraintes de mobilité et difficultés d'accès aux soins dans la métropole d'Oran », Territoire en Mouvement, $\mathrm{n}^{\circ}$ 4, p. 3-16.

REBOUHA F. [2010], Transport, mobilité, et accès aux services des populations défavorisées : Le cas des habitants des grandes périphéries, Thèse de doctorat en Sciences, spécialité architecture, option urbanisme, université des Sciences et de la technologie d'Oran, 280 p. + Annexes: http://tel.archives-ouvertes.fr/tel-00595990/fr/

REBOUHA F., POCHET P. [2009], « Mobilité et accès à la ville pour le travail des populations pauvres du périurbain d'Oran », Cahiers de la Méditerranée, n ${ }^{\circ} 78$, p. 335-353.

SIKIROU S. [2008], «Pauvreté et accessibilité des ménages urbains aux biens et services publics à Ouagadougou et de Bobo Dioulasso », in KouAssi B. (dir.), Pauvreté des ménages et accès aux soins de santé en Afrique de l'ouest: Burkina Faso, Côte-d'Ivoire, Ghana, Mali et Togo, Paris, Karthala, p. 51-102.

SITRASS [2004], Mobilité et pauvreté urbaine à Douala, Rapport Final, Lyon-Arcueil, Rapport pour la Banque mondiale dans le cadre du SSATP, 154 p.: http://www4.worldbank.org/afr/ssatp/Resources/PapersNotes/Douala.pdf (page consultée le 29 octobre 2010).

ZoungRAnA C.M., TOKIndANG J., MARCOUX R., KonATE M. [1998], «La trajectoire scolaire des filles à Bamako. Un parcours semé d'embûches », in LANGE M.-F. (dir.), L'École et les filles en Afrique. Scolarisation sous conditions, Paris, Karthala, p. 167-196. 